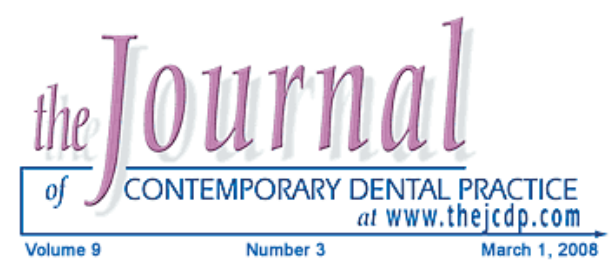

\title{
Treatment of Painful Post-traumatic Peripheral Neuropathy with Capsaicin in an Edentulous Patient with Extreme Resorption in the Mental Region: A Case Report
}

\section{Therezinha Pastre, DDS, MSc; Fornanda Faot, DDS, MSc, PhD; \\ Fernando H. Westphalen, DDS, MSc, PhD;}

Rosena Santos da Rosa, DDS, MSc, PhD

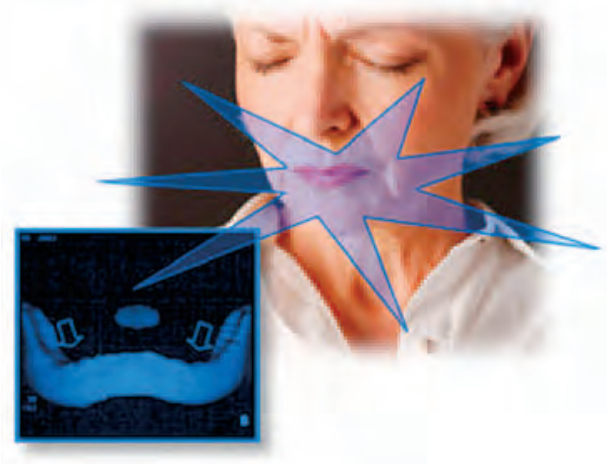

Abstract

Aim: The aim of this report is to present a clinical case of extensive alveolar resorption in the mental region of the mandible of a complete denture wearer for which capsaicin was used for alleviation and remission of posttraumatic painful peripheral neuropathy.

Background: Resorption of the residual ridge occurs in a chronic and progressive manner, altering the configuration of the ridge and the mucosa covering it and subsequently compromising the stability of the complete denture prosthesis. Individual variability associated with such factors as gender, age, edentulous period, nutrition, excessive load and compression on the ridge, parafunctional habits, occlusal instability, and maladaptation of the prosthesis may have a role in the rate and manner of bone loss.

Report: A 62-year-old woman, edentulous at the age of 30 , presented with complaints of pain and spontaneous "electrical shocks" in the mucosa under her mandibular complete denture. The pain increased while chewing approximately two years ago due to the pressure of this denture on her supporting tissues. The patient indicated the level of pain corresponded to a score of ten out of ten on the Visual Analogical Scale (VAS). Treatment included denture replacement and topical application of capsaicin gel using a custom tray technique.

Summary: The review of the literature and the results obtained from the management of this case have served to emphasize how much neuropathic pain research has demonstrated the complexity of its neurophysiologic mechanism due to the lack of association with any somatic manifestation.

(c) Seer Publishing 
During the fabrication of a new lower denture the topical application of capsaicin was shown to be effective in the relief and remission of the painful symptoms of the neuropathy as of this writing.

Keywords: Alveolar resorption, capsaicin, neuropathic pain, mental nerve exposure, neuropathic pain relief

Citation: Pastre T, Faot F, Westphalen FH, da Rosa RS. Treatment of Painful Post-traumatic Peripheral Neuropathy with Capsaicin in an Edentulous Patient with Extreme Resorption in the Mental Region: A Case Report. J Contemp Dent Pract 2008 March; (9)3:106-113.

\section{Introduction}

Resorption of the residual ridge after tooth extraction occurs in a chronic and progressive manner. This anatomical change alters the configuration of the ridge and its mucosal covering making it difficult to fabricate a stable complete prosthesis. The index and form of alveolar resorption varies in the dental arches and varies with individual denture wearers, due to the following factors: ${ }^{1-2}$

- Loss of the periodontal membrane

- Surgical exposure of the alveolar bone

- Duration of edentulism

- Excessive load and compression on the alveolar ridge

- Bruxism

- Occlusal instability

- Prolonged use of the prosthesis

- Systemic diseases such as osteoporosis

When a condition of accentuated bone resorption is observed in both dental arches, the tendency for surface exposure of the nerves in these regions is increased and the nerves become more susceptible to continuous traumas of peripheral origin. Such a localized neurosensory disturbance may be caused by pressure on the mental foramen or the mental nerve generated by a complete denture. ${ }^{3}$ A neural injury may be a consequence of this problem that can lead to the development of peripheral sensitization of nociceptors inducing a sustained pain sensation (hyperalgesia, allodynia, pain spread). ${ }^{4}$ In addition, specific mechanisms of action can lead to a chronic peripheral nociceptor irritability and develop central neural changes responsible for the initiation of a post-traumatic painful peripheral neuropathy, commonly known as neuropathic pain. ${ }^{5}$

Generally, neuropathic pain from a post-traumatic painful peripheral neuropathy is characterized by a debilitating tactile allodynia or an excruciatingly painful and persistent response to a normally innocuous mechanical stimulus. ${ }^{6}$ Such pain is initiated by a primary lesion or dysfunction in the peripheral nervous system. ${ }^{7}$ At the orofacial level, neuropathic pain can result from nerve trauma associated with pressure, complete or partial deafferentation or amputation, infection, or metabolic disturbance. ${ }^{8}$ It is difficult to diagnose because clinical changes in the tissues involved are rare. ${ }^{9}$ The clinical symptoms include burning, stabbing, dormancy, tingling, or bizarre sensations. ${ }^{10}$ Since the pain does not manifest itself in all patients with similar lesions it is likely genetic factors are involved in its occurrence. ${ }^{11}$

The mechanism of hyperexcitability of primary afferent neurons, the occurrence of ectopical foci of potential action in the peripheral nerve fibers and central pathways, as well as the abnormal activity of the central processing units of the sensory afferences are critical to understanding the genesis of neuropathic pain and its modalities like post-traumatic painful peripheral neuropathy. This article presents a literature review with respect to the pathophysiology of neuropathic pain and its treatment with the use of capsaicin, along with a description of a clinical case including the signs, symptoms, and treatment of posttraumatic painful peripheral neuropathy.

\section{Review of the Literature}

\section{Pathophysiology of Neuropathic Pain}

The integrity of the nociceptive pathways in the peripheral nervous system (PNS), the central nervous system (CNS), the processing centers, and pain modulation of the CNS must be intact for the nociceptive phenomenon to be processed normally. If there is a lesion involving nerve fibers, the potential of an immense rise in amplitude occurs in the primary afferents for a few seconds. 
The proximal stump of sectioned axons is sealed and the adjacent myelin sheath and the axons degenerate to the extent of a few millimeters.

After some time, groups of axons emerge from the terminal bulbs and under suitable conditions reach the nerve terminals in the tissues. The growing nerve fibers generate action potentials spontaneously and are sensitive to mechanical stimulation and to the effects of adrenalin. In time, the restoration of the normal functions of nociceptive receptors occurs. ${ }^{12}$

However, when the growth of the nerve is blocked, the formation of a neuroma occurs. When the lesion is partial and regeneration is blocked, microneuromas arise at different intervals. Ectopic activity appears as the lesion increases during the first week and then progressively declines. The ectopic potentials of neuromas are more abundant when there is tissue ischemia, raised extracellular potassium concentration, and the accumulation of peptides. In peripheral neuropathies there is disseminated regeneration and degeneration along the fibers which frequently results in the occurrence of spontaneous pain. ${ }^{13}$

The accumulation of potassium and other ions in the region of the neuroma may generate depolarization in the neighboring nerve fibers. Ephaptic currents seem to occur in amputation neuromas and in degenerating nerve fibers. Ectopic potentials may also appear when the action potentials of the primary afferents are prolonged beyond the absolute refractory period generating re-excitation of the neuronal membrane. A lesion of peripheral nerves generates anatomical changes in the nuclei of the ganglion cells of sensitive nerves and in the central projection of nerve roots. ${ }^{14}$

Thus, neuronal hyperexcitability is due to the appearance of pace makers in the regenerating peripheral nerves and results in a change in the permeability of the axonal membrane as well as in the number, distribution, and kinetics of the sodium, calcium, and potassium channels. Cell units predominantly excited by nociceptive stimuli also begin to respond to low intensity stimuli in cases of deafferentation. ${ }^{15}$

A lesion of sensitive spinal and trigeminal roots results in degeneration of the axons and of their projections in the CNS and after a few months, an increase in the distribution of the primary afferents. Thus, lesions of the PNS involve functional alterations in the second order neurons of the posterior stump of the spinal medulla. Later, functional abnormalities occur in the thalamus. ${ }^{14-15}$

There are therapies for the relief of orofacial pain of this nature in patients with extremely reabsorbed residual ridges reported in the dental literature. These include the following:

- Internal relief of removable prostheses

- Amputation and lateralization of the inferior alveolar nerve ${ }^{16}$

- Rehabilitation with implants to reduce contact in the mental nerve ${ }^{17}$

- Application of soft resins ${ }^{18}$

- Surgical lowering of the mental foramen ${ }^{3}$

- Use of topical medications such as $0.025 \%$ capsaicin ${ }^{4,19-20}$

\section{Capsaicin}

Capsaicin (the substance responsible for the burning sensation produced by hot chili peppers) is a molecule capable of interacting with nociceptors activated by heat stimulus, called vanilloid receptors, whose structure has not yet been completely elucidated. Certain nerve terminals open sodium channels, generating an electrical impulse that is propagated along the nervous system when they are exposed to the capsaicin molecule. ${ }^{21-23}$

Generally, the vanilloids have a two-phase action in sensitive peripheral nerves; an initial excitative phase (manifested as pain and/or neurogenic inflammation) followed by an analgesic phase of loss of the receptor sensitivity known as de-sensitization. ${ }^{24-25}$

Peripheral sensorial neurons treated with capsaicin release $P$ substance, the neurotransmitter of choice in the sensorial trajectory of pain, causes an over-stimulation and sensation of burning pain. ${ }^{26}$ The amount of $P$ substance in the primary afferent nerve terminals of sensorial neurons may be reduced by the action of capsaicin or by the possibility of the capsaicin receptor/ionic channel having been desensitized with progressive doses. ${ }^{26}$ 
In this context a molecule causing pain may also alleviate it. Such is the case with capsaicin which is widely used as an analgesic for the treatment of arthritis and as a means of helping to quantify alterations in neuronal sensitivity in this pathology. ${ }^{23,27}$

The explanation for the pain suppression mechanism is the continual exposure of a nociceptor to capsaicin literally destroys this cell as the continual influx of calcium ions into the permanently open membrane drastically alters the functions of the cell, leading to its annihilation. ${ }^{4,23,28}$

\section{Clinical Case Report}

\section{Diagnosis}

A 62-year-old woman, edentulous at the age of 30 , presented with complaints of pain and spontaneous "electrical shocks" in the mucosa under her mandibular complete denture. The pain increased while chewing approximately two years ago due to the pressure of this denture on her supporting tissues. The patient indicated the level of pain corresponded to a score of ten out of ten on the Visual Analogical Scale (VAS). A score of ten is considered to be the worst, unbearable pain capable of affecting a person's daily routine.

Upon clinical examination there was no visible tissue alteration, but palpation of the mental region on both sides of the mandible elicited pain and a "shock" sensation. The panoramic and tomographic examinations revealed extensive mandibular bone resorption (Figures 1 and 2). However, no bone and mucosa alterations were found that justified the presence of the symptoms.

The preliminary diagnosis was post-traumatic painful peripheral neuropathy probably triggered by physiological resorption resulting from the process of aging, total loss of teeth, and accelerated by the use of a complete prosthesis in cross bite (false Class III) for three years (Figure 3). The lower denture was adjusted several times to provide internal relief in the mental foramen region without any reduction of the painful symptoms.

\section{Treatment \\ The patient was treated with a topical application of $0.025 \%$ capsaicin gel placed along with $10 \%$ xylocaine on the painful region five times a day for ten minutes for 30 days. The gel was delivered and kept in place with the help of a custom acrylic tray fabricated with relief areas in the mental nerve (Figures 4 and 5). Replacement of the complete denture was also necessary because internal relief of the old denture was performed unsuccessfully several times in an attempt to relieve the contact of the denture with the mental nerve.}

The therapeutic protocol was simple to implement and was accepted by the patient. Relief of the painful symptoms was measured using the VAS

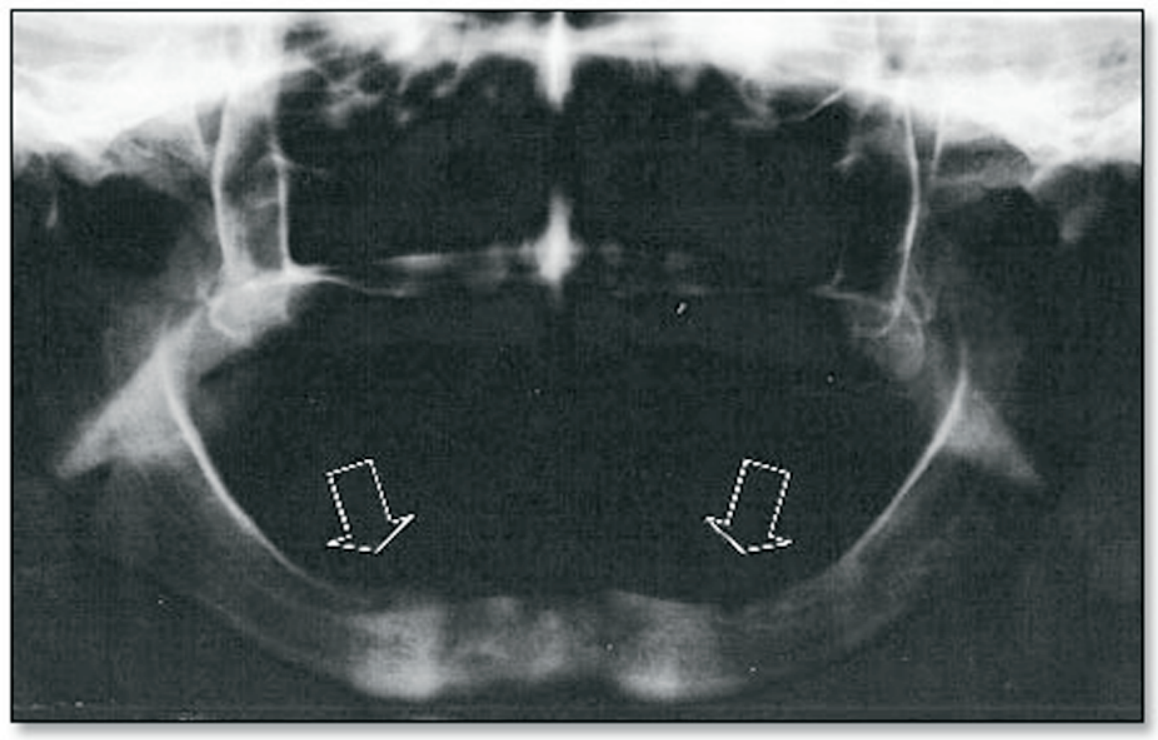

Figure 1. Panoramic radiograph showing extensive reabsorption of the lower alveolar ridge. 

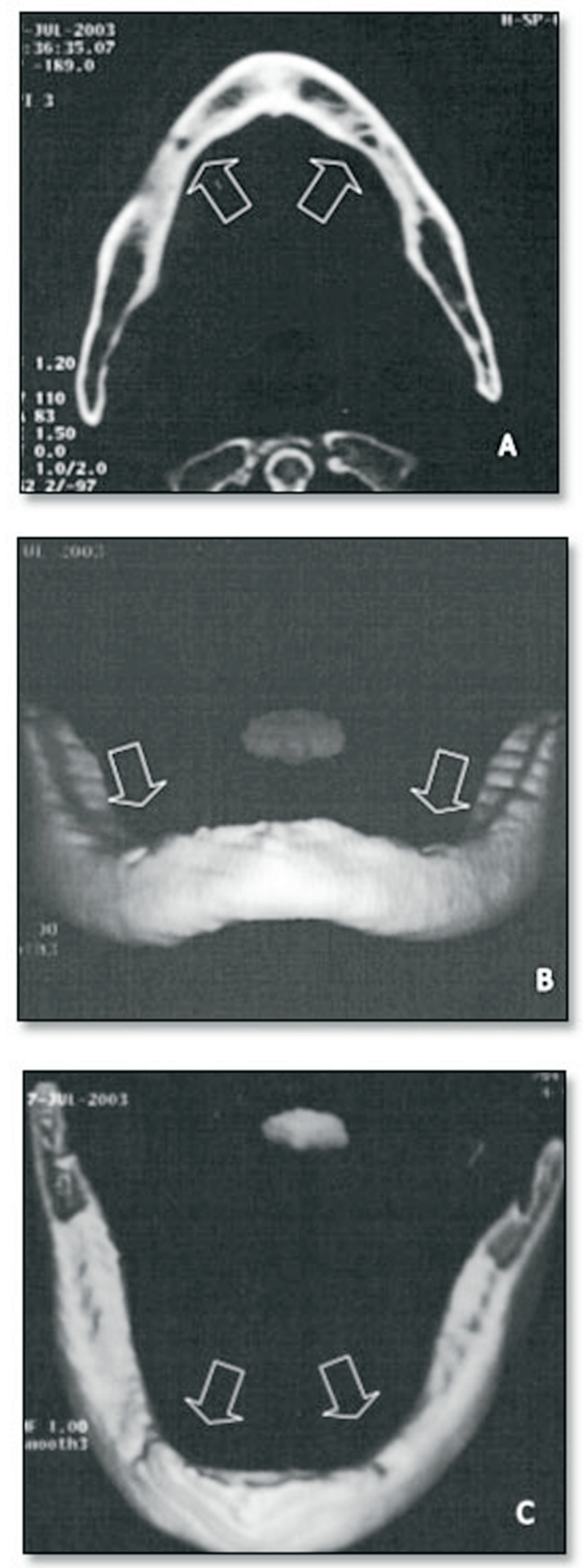

Figure 2. A, B, C Tomography confirming extensive re-absorption of the lower alveolar ridge.

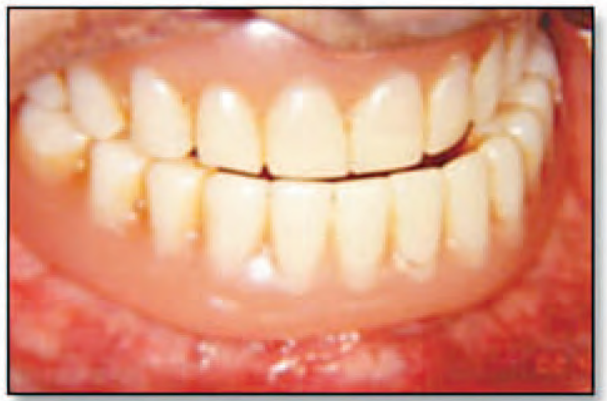

Figure 3. Old complete prosthesis with a cross bite.

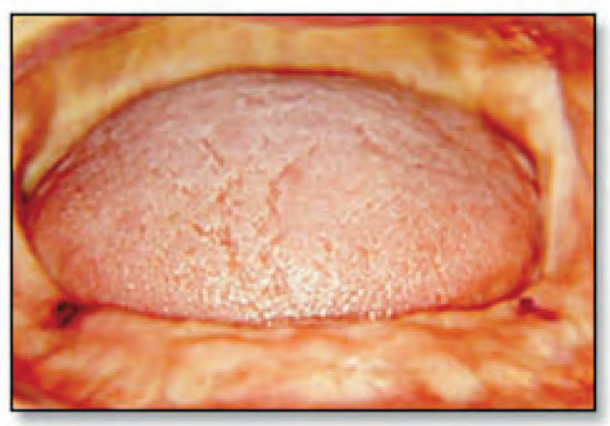

Figure 4. Intraoral exam demarcating the points of pain.

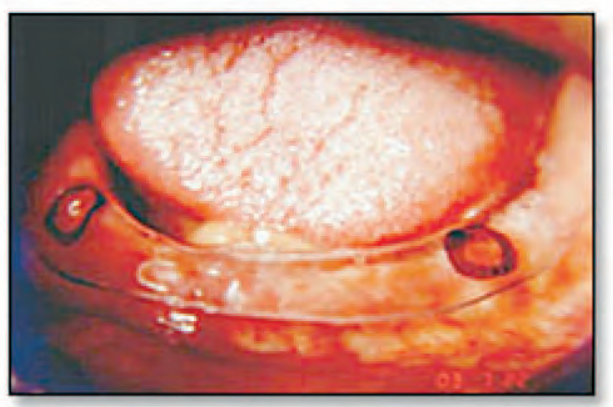

Figure 5. Topical application of gel with the aid of a special tray with areas of relief in the areas of the mental nerves. 
used previously. The following scores were

recorded during treatment:

- Week 1: VAS score of 6

- Week 2: VAS score of 4

- Week 3: VAS score of 2

- 30 days and beyond: VAS score of 0

Pain remission was achieved within 30 days after which new complete dentures were fabricated. Periodic examinations were carried out at three, six, nine, 12 and 18 month intervals revealed the patient to be asymptomatic.

\section{Discussion}

Residual ridge resorption affects the anatomicalphysiological relation between the mental nerve and the bone tissue. The mental foramen region covered only by gingival mucosa predisposed this edentulous patient to greater susceptibility to pain in the presence of trauma or compression by the mandibular complete denture. This case report and review of the literature presented the diagnosis of localized orofacial pain caused by the development of extensive mandibular alveolar resorption and treatment consisting of medication with capsaicin and subsequent denture replacement.

The neurogenic inflammation and the cascade of events followed by the neural injury that originates neuronal deafferentation have been described and have become a useful model for understanding painful neuropathyies. Inflammatory neuropeptides (Substance P) and prostaglandins (PGE2) may be released from the primary afferent nociceptors and sympathetic postganglionic neurons activating nearby receptors and triggering the spread of activation. ${ }^{12,14,29}$ These mechanisms may explain the clinical response of some patients with neuropathic pain to topical medications such as: non-steroid anti-inflammatory drugs, lidocaine, and capsaicin. ${ }^{4}$
Capsaicin exercises a strong pharmacological action on the peripheral nervous system and on the CNS. This affects the ionotropical vanilloid receptors of primary afferent neuron Type $C$ fibers by reducing the peripheral neural sensitivity of the area sensitized by the painful heat stimulus. ${ }^{21-22}$

The advantages of the pain control technique used are providing pain relief without side effects and the ease of application by the patient using the custom tray to place and contain the capsaicin gel directly over the affected region without the need for professional assistance. This was continued until the pain was resolved then new dentures were fabricated for functional reasons.

Treatment protocols should be continuously evaluated as needed since the topical application of $0.03 \mathrm{mg} / \mathrm{ml}$ capsaicin doses for 30 minutes was found to be unable to generate a painful local stimulus in hypersensitized patients such as those with rheumatoid arthritis. ${ }^{30}$ The disadvantage of this protocol is the lack of a suitable measurement for quantifying improvement in pain symptoms. The VAS is based solely on the patient's subjectivity since there are no diagnostic tests to quantify the intensity of pain.

\section{Conclusion}

The review of the literature and the results obtained from the management of this case has served to emphasize how much neuropathic pain research has demonstrated the complexity of its neurophysiologic mechanism due to the lack of association with any somatic manifestation.

Before the fabrication of a new lower denture, the topical application of capsaicin was shown to be effective in the relief and remission of the painful symptoms of the neuropathy as of this writing. 


\section{References}

1. Atwood DA. Some clinical factors related to rate of resorption of residual ridges. J Prosthet Dent $1962 ; 12: 441-450$.

2. Tallgren $A$. The continuing reduction of the residual alveolar ridges in complete denture wearers: A mixed-longitudinal study covering 25 years. J Prosthet Dent 2003, 89:427-435.

3. Wismeijer D, van Waas MAJ, Vermeeren JIJF, Kalk W. Patients' perception of sensory disturbances of the mental nerve before and after implant surgery: a prospective study of 110 patients. $\mathrm{Br} \mathrm{J}$ Oral Maxillofac Surg. 1997; 35:254-259.

4. Padilla M, Clark GT, Merrill RL. Topical medications for orofacial neuropathic pain: a review. J Am Dent Assoc. 2000; 131:184-95.

5. Bennett GF. Neuropathic pain in the orofacial region: clinical and research challengers. J Orofac Pain 2004; 18:281-286.

6. Henry LJ. Future basic science directions into mechanisms of neuropathic pain. J Orofac Pain 2004; 18:306-310.

7. McNeill C, Dubner R. What is pain and how do we classify orofacial pain. In: Lund JP, Lavigne GJ, Dubner R, Sessle BJ. Orofacial Pain. From basic science to clinical management. Illinois: Quintessence, 2001: 8-9.

8. Max M. Management of neuropathic pain. In: Lund JP, Lavigne GJ, Dubner R, Sessle BJ. Orofacial Pain. From basic science to clinical management. Illinois: Quintessence, 2001: 259-269.

9. Iwata K, Tsuboi Y, Shima A, Harada T, Ren K. Central Neuronal changes after nerve injury: neuroplastic influences of injury and aging. J Orofac Pain 2004; 18:281-286.

10. Tasker RR, Emmers R. Patterns of somesthetic projection in S I and S II of the human thalamus. Confin Neurol. 1967; 29(2):160-2.

11. Willis WD. The origin and destination of pathways involved in pain transmition. In: Wall, P.D. \& Melzack, R. Textbook of Pain. Edinburgh Churchill Livingstone, 1989: 112-127.

12. Yaksh TL, Chaplan SR. Physiology and pharmacology of neuropathic pain. Anesthesiology Clinics of North America 1997; 15 (2):335-352.

13. Devor M. Neuropathic pain and injured nerve - peripheral mechanisms. Br Med Bull 1991; 47:619-630.

14. Asbury AK, Fields HL. Pain due to peripheral nerve damage; an hypothesis. Neurology 1984; 34:1587-1590.

15. Johnson BW, Parris WCV. Mechanisms of Neuropathic Pain. In Current Review of Pain, Raj PP, (ed.) Current Medicine, 1994.

16. Peleg M, Mazor Z, Chaushu G, Garg AK. Lateralization of the inferior alveolar nerve with simultaneous implant placement: a modified technique. Int J Oral Maxillofac Implants 2002; 17(1):101-6.

17. Stellingsma K, Raghoebar GM, Meijer HJA, Stegenga B. The extremely resorbed mandible: a comparative prospective study of 2-year results with 3 treatment strategies. Int J Oral Maxillofac Implants 2004; 19:563-577.

18. Williamson RT. Clinical application of a soft denture liner: a case report. Quintessence Int. 1995 Jun;26(6):413-8.

19. Epstein JB, Marcoe JH. Topical application of capsaicin for treatment of oral neuropathic pain and trigeminal neuralgia. Oral Surg Oral Med Oral Pathol 1994; 77:135-40.

20. Baad-Hansen L, Jensen TS, Svensson P. A Human model of intraoral pain and heat hyperalgesia. J Orofac Pain 2003; 17:333-340.

21. Dray A. Neuropharmacological mechanisms of capsaicin and related substances. Biochem Pharmacol. 1992 Aug 18;44(4):611-5.

22. Dray A. Mechanism of action of capsaicin-like molecules on sensory neurons. Life Sci. 1992; 51(23):1759-65.

23. Caterina MJ, Schumacher MA, Tominaga M, Rosen TA, Levine JD, Julius D. The capsaicin receptor: a heat-activated ion channel in the pain pathway. Nature 1997; 389(23):816-824. 
24. Sweitzer SM, Peters MC, Ma JY, Kerr I, Mangadu R, Chakravarty S, Dugar S, Medicherla S, Protter AA, Yeomans DC. Peripheral and central p38 MAPK mediates capsaicin-induced hyperalgesia. Pain 2004; 111:278-285.

25. Malmberg AB, Mizisin AP, Calcuta NA, von Stein T, Robbins WR, Bley KR. Reduced heat sensitivity and epidermal nerve fiber immunostaining following single applications of a high-concentration capsaicin match. Pain 2004; 111:360-367.

26. Schmid G, Carita F, Bonanno G, Raiteri, M. NK-3 receptors mediate enhancement of substance $\mathrm{P}$ release from capsaicin-sensitive spinal cord afferent terminals. $\mathrm{Br} \mathrm{J}$ Pharmacol 1998; 125(4):621-626.

27. Morris HV, Cruvys SC, Kidd BL. Characterisation of capsaicin-induced mechanical hyperalgesia as a marker for altered nociceptive processing in patients with rheumatoid arthritis. Pain 1997; 71:179-184.

28. Oh U, Hwang SW, Kim D. Capsaicin activates a nonselective cation channel in cultured neonatal rat dorsal root ganglion neurons. J Neurosci 1996; 16:1659-1667.

29. Levine JD, Fields HL, Basbaum Al. Peptides and the primary afferent nociceptor. J Neurosci 1993; 13:2273-2286.

30. Morris HV, Cruvys SC, Kidd BL. Characterisation of capsaicin-induced mechanical hyperalgesia as a marker for altered nociceptive processing in patients with rheumatoid arthritis. Pain 1997; 71:179-184.

\section{About the Authors}

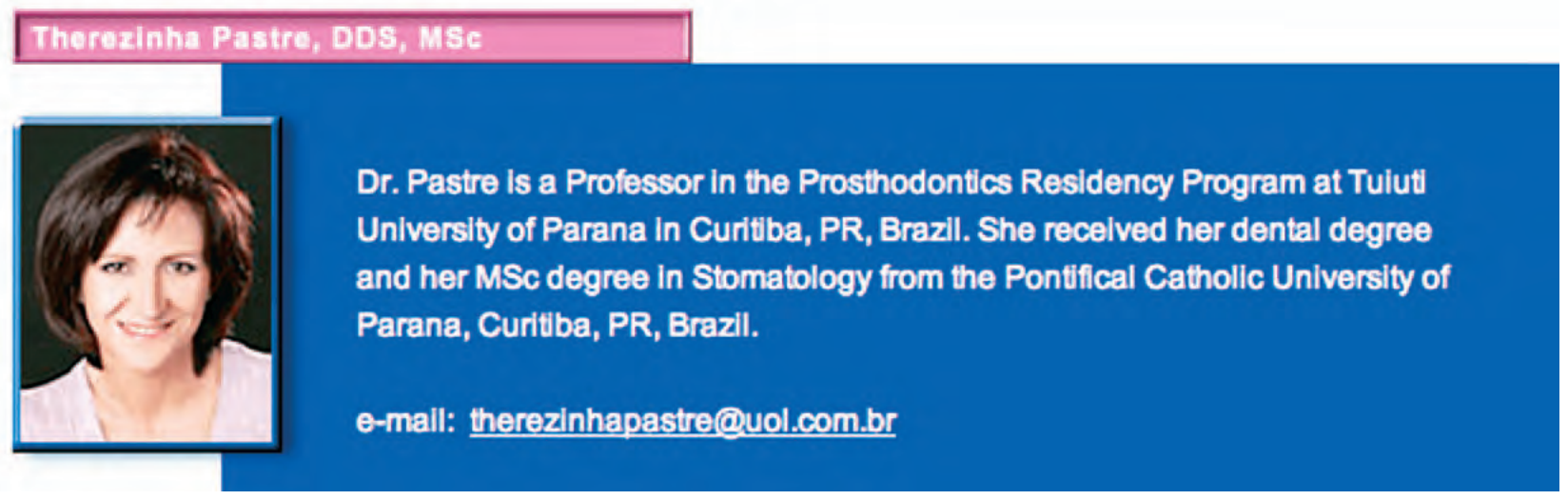

\section{Fornanda Faot, DDS, MSc, PhD}

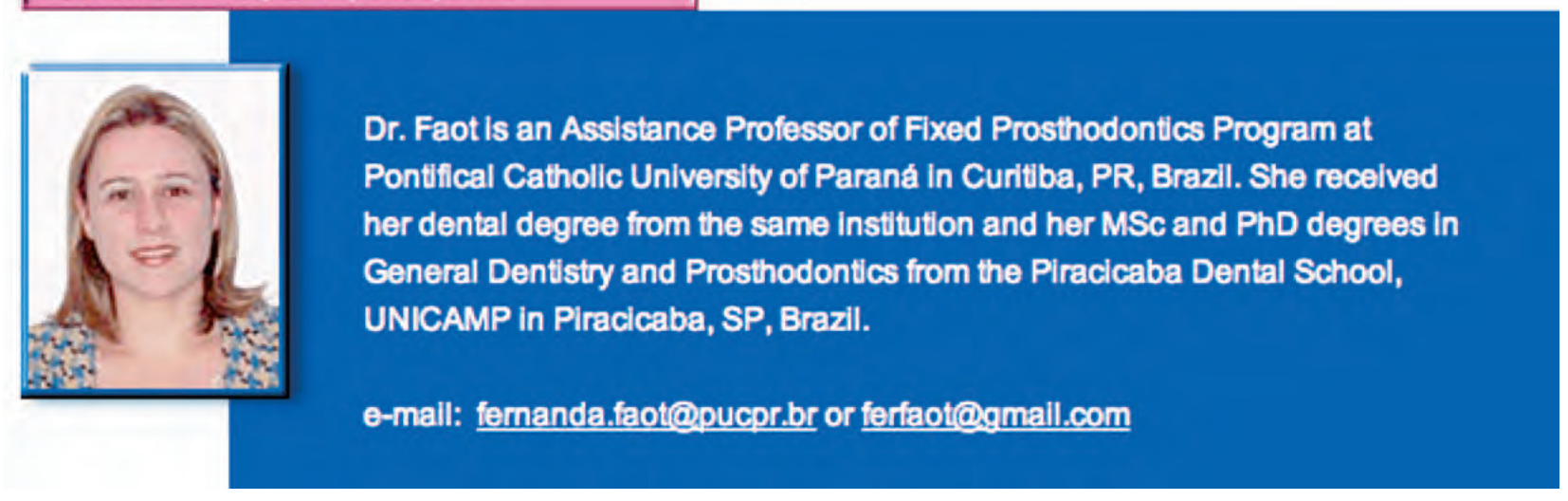




\section{Fornando H. Westphalen, DDS, MSc, PhD}

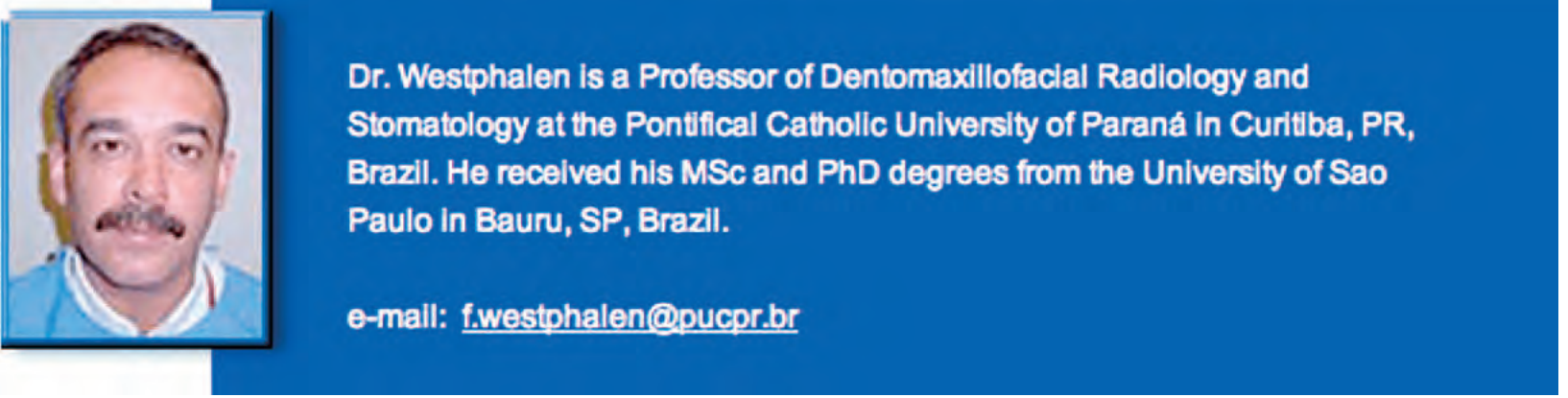

\section{Rosena Santos da Rosa, DDS, MSc, PhD}

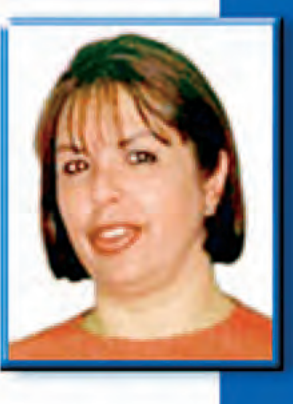

Dr. Santos da Rosa is an Adjunct Professor of Fixed Prosthodontics Program at Pontifical Catholic University of Parana in Curitiba, PR, Brazil. She recelved her dental degree from the same Institution and her MSc and PhD degrees from the Piracicaba Dental School, UNICAMP in Piracicaba, SP, Brazil.

e-mail: rosena.rosa@pucpr.pr, rosenasr@uol.com.br 